\title{
Les besoins des patients atteints d'un cancer avancé
}

\author{
By Margaret I. Fitch
}

\section{Sommaire}

De nombreux changements surviennent chez la personne atteinte d'une maladie fatale. Ces changements dépassent le simple domaine physique et incluent des conséquences psychosociales, pratiques et spirituelles. Si la maladie s'aggrave, l'impact de ces changements est intensifié. Les infirmières qui soignent des patients atteints d'un cancer avancé doivent pouvoir effectuer des interventions appropriées si elles veulent favoriser l'adaptation de ces derniers. Cet article dégage les besoins des patients atteints d'un cancer avancé et souligne des approches qui pourraient être utilisées par les infirmières en oncologie dans la prise en charge de ces patients. Les données proviennent de plus d'une décennie d'entrevues auprès de patients sur leur expérience avec le cancer, des écrits sur les besoins des patients et de consultations avec les prestataires de soins. La perspective globale est de voir dans les êtres humains des entités biopsycho-socio-spirituelles. L'adoption de cette perspective a des répercussions sur l'évaluation des patients atteints d'un cancer avancé et sur les interventions liées à leur prise en charge.

Lorsque je regarde en arrière, que je pense à tout le temps que j'ai été malade, il n'y a pas une partie de ma vie qui n'a pas été touchée. Pas une seule partie. Tout est maintenant différent. (un patient atteint d'un cancer de la prostate, environ 75 ans)

De nombreux changements surviennent chez la personne atteinte d'une maladie mortelle. Ceux-ci ne se limitent pas aux changements physiques dus à la maladie et à ses symptômes mais également aux traitements et à leurs effets secondaires. Les changements sont également sociaux, psychologiques et spirituels, pour la personne atteinte et pour les membres de sa famille. Tous en ressentiront l'impact à un degré ou à un autre ainsi que la détresse émotionnelle qui en découle. De plus, si la menace d'une mort causée par la maladie devient une réalité et que la mort est imminente, les émotions s'amplifient et peuvent devenir accablantes (McLean, 1993). Les infirmières qui soignent ces patients doivent pouvoir effectuer des interventions appropriées si elles veulent les aider à s'adapter à ce qui leur arrive et à la détresse émotionnelle ainsi engendrée.

Cet article dégage les besoins des patients atteints d'un cancer avancé et souligne des approches clés qui pourraient être utilisées par les infirmières afin de s'assurer que les besoins de leurs patients sont satisfaits. Les données proviennent de plus d'une décennie d'entrevues auprès de patients sur leur expérience avec le cancer, des écrits sur les besoins des patients, de la lecture de rapports de recherche sur les besoins et les perspectives des patients, et de consultations avec un large éventail de prestataires de soins. J'ai choisi quelques-unes de mes citations favorites tirées des entrevues avec les patients afin d'illustrer les principales perspectives pour chaque domaine. La perspective globale est de voir dans les êtres humains des entités bio-psychosocio-spirituelles, et non seulement des êtres physiques. L'adoption de cette perspective a, pour l'infirmière, des implications sur la manière dont les soins en oncologie sont prodigués.

\section{Les besoins des êtres humains}

En tant qu'êtres humains, nous avons tous des besoins physiques, psychologiques, sociaux, émotionnels, spirituels et pratiques ainsi que des besoins d'information (Ferrell, 1996). Nous essayons de satisfaire ces besoins tous les jours : se reposer, dormir, manger, travailler, jouer et développer des mécanismes d'adaptation. Toutefois, lorsque la maladie frappe, il peut nous être impossible de satisfaire nos besoins quotidiens de la même façon. De nouvelles demandes peuvent surgir et l'anxiété et l'incertitude font leur apparition (voir la figure 1). Tout à coup, nous pouvons nous sentir vulnérables, penser que les choses sont incontrôlables et croire que nous ne pouvons pas faire face à la situation. Pour satisfaire nos besoins, il est possible que nous devions chercher de nouveaux renseignements, apprendre de nouvelles compétences ou rechercher l'aide d'autrui.

Margaret I. Fitch, RN, PhD, est Directrice de l'Unité de recherche psychosociale et comportementale, Centre régional de cancérologie de Toronto-Sunnybrook. Pour tout contact : marg.fitch@sw.ca 
Certains individus atteints d'une maladie peuvent mobiliser leurs propres ressources et leur réseau social et obtenir l'aide dont ils ont besoin lorsqu'ils ont accès à l'information, au soutien et à la communication appropriés. Ces individus trouvent le moyen de composer avec les nouvelles demandes provoquées par la situation, d'autres non. Ces derniers continuent d'éprouver des difficultés et une détresse émotionnelle, et leurs besoins sont toujours insatisfaits (Société canadienne du cancer, 2004). Ils nécessitent un soutien supplémentaire et des interventions spécialisées afin de pouvoir s'adapter à ce qui leur arrive.

Lorsqu'elles prodiguent des soins aux malades, les infirmières en oncologie doivent être en mesure de faire une évaluation des types de difficultés auxquels ils font face, du degré de détresse émotionnelle engendré par ces difficultés et déterminer si les patients ont les ressources nécessaires pour s'adapter à la situation. Fondées sur une évaluation complète, des interventions peuvent être personnalisées afin de satisfaire un groupe particulier de besoins chez la personne.

\section{Les besoins physiques}

Qu'est-ce qui est le plus pénible? Eh bien, je dirais que ce n'est pas une chose en particulier. Ce sont tous les symptômes : la douleur, la fatigue, la nausée, l'insomnie... c'est tout ça ensemble. (une patiente atteinte d'un cancer de l'ovaire, environ 65 ans)

À mesure que la maladie progresse, les symptômes et les effets secondaires des traitements augmentent et sont plus prononcés. Bien que les symptômes spécifiques varient selon le type et le stade particuliers de la maladie (voir le tableau 1), les symptômes les plus communs incluent la douleur, la perte d'appétit, la fatigue, la faiblesse, la perte de poids, la constipation, la gêne respiratoire, le désarroi, la nausée, les vomissements, la toux, et la sécheresse ou les ulcérations de la bouche (Fitch et Steele, 2003; Ashbury, Findley, Reynolds et McKerralehen, 1998). Toutefois, il est rare que les patients n'éprouvent qu'un seul symptôme en particulier. L'expérience la plus commune, chez les patients, est de présenter un ensemble - ou un groupe - de symptômes qui s'influencent les uns les autres. Par exemple, la douleur sera exacerbée si le sommeil est perturbé ou si la fatigue augmente. Un des défis des soins cliniques est de comprendre comment le patient vit ces ensembles ou groupes de symptômes et d'offrir des interventions appropriées (Dodd et al., 2003).

La détresse émotionnelle des patients augmente si l'on ne considère pas leurs symptômes et, par conséquent, leur qualité de vie en est diminuée (Singer, Martin et Kelner, 1999). Selon des recherches récentes, un niveau élevé de détresse émotionnelle résultant d'une mauvaise gestion des symptômes est lié à la diminution de la survie, tout particulièrement chez les patients atteints d'un cancer avancé (Edmonds, Karlsen, Khan et Addington-Hall, 2001). Pour ces motifs, un des buts essentiels de la prestation des soins aux personnes atteintes d'un cancer avancé est de soulager la douleur et les autres symptômes pénibles. Malheureusement, certains patients déclarent qu'à leur avis, leurs symptômes n'ont pas été convenablement traités (voir le tableau 2).

Les médecins et les infirmières devront peut-être essayer différentes approches ou interventions afin de trouver la manière la plus efficace de maîtriser les effets secondaires et les symptômes (Preston et Cunningham, 1998). Une surveillance et une évaluation continue du confort physique du patient sont importantes afin de déterminer si certaines interventions sont utiles et d'y apporter des changements appropriés si elles ne sont pas efficaces. Si l'intervention initiale ne fonctionne pas, un ajustement rapide et de nouvelles interventions sont d'une importance capitale. Les équipes cliniques ont conclu que l'utilisation de listes de contrôle normalisées pour l'évaluation et la documentation des symptômes, de pair avec des protocoles prédéfinis pour les symptômes, favorisait un ajustement rapide (Sarna, 1998).

\section{Les besoins d'information}

Vous savez, ce qui a fait toute la différence pour moi, c'était de pouvoir obtenir des renseignements, des renseignements que je pouvais comprendre. C'était comme si quelqu'un avait allumé la lumière dans une pièce noire. (une survivante du cancer du sein, environ 55 ans)

Lorsque la maladie s'aggrave, les patients veulent comprendre ce qui arrive à leurs corps, et veulent participer aux décisions relatives à la manière dont ils vont jouir de leurs derniers jours (Fitch, Noble, Friedman, MacDonald, Morrison, Munnoch et al., 2004). Toutefois, il est important qu'ils aient accès à des renseignements pertinents s'ils veulent participer aux discussions et prendre des décisions éclairées.

Les patients jugent qu'il est très important d'avoir accès à des renseignements sur leurs conditions physiques, leur maladie, les options de traitement ainsi que leurs effets secondaires (Fitch, Gray et Franssen, 2000; Fitch et Plewes, 2004) (voir le tableau 3). Plusieurs patients disent qu'être atteint d'une maladie signifie qu'ils entrent dans un monde inconnu où un langage inhabituel est utilisé. Ils

Tableau 1. Symptômes physiques signalés par les patients atteints d'un cancer avancé *

\begin{tabular}{|l|l|l|}
\hline & \multicolumn{2}{|l|}{$\begin{array}{l}\text { Proportion des patients } \\
\text { signalant les symptômes }\end{array}$} \\
\hline Symptôme physique & $\begin{array}{l}\text { Patients atteints } \\
\text { d'un cancer du } \\
\text { poumon N=86 }\end{array}$ & $\begin{array}{l}\text { Patients en soins } \\
\text { palliatifs N=63 }\end{array}$ \\
\hline Manque d'énergie & 68,6 & 80,9 \\
\hline Essoufflement & 48,8 & 57,1 \\
\hline Toux & 46,5 & 31,7 \\
\hline Douleur & 33,7 & 46,0 \\
\hline Perte d'appétit & 30,2 & 20,6 \\
\hline Nausée/vomissements & 15,1 & 9,5 \\
\hline$*$ (Fitch et Steele, 2003) & \\
\hline
\end{tabular}

Tableau 2. Patients atteints de cancer indiquant qu'ils n'avaient pas reçu une assistance adéquate pour leurs symptômes

\begin{tabular}{|l|l|l|}
\hline Symptômes & $\begin{array}{l}\text { Patientes atteintes } \\
\text { d'une récidive du } \\
\text { cancer de l'ovaire } \\
\text { N=95* Proportion } \\
\text { signalant une } \\
\text { assistance } \\
\text { inadéquate }\end{array}$ & $\begin{array}{l}\text { Patients d'un cancer } \\
\text { de la prostate (de } \\
\text { divers stades) } \\
\text { N=621** Proportion } \\
\text { signalant une } \\
\text { assistance } \\
\text { inadéquate }\end{array}$ \\
\hline Effets secondaires & 23,0 & 22,0 \\
\hline $\begin{array}{l}\text { Perturbation } \\
\text { du sommeil }\end{array}$ & 43,0 & Non signalé \\
\hline $\begin{array}{l}\text { Selles difficiles } \\
\text { ou incontinence }\end{array}$ & 65,0 & 35,0 \\
\hline Douleur & 15,0 & 19,0 \\
\hline $\begin{array}{l}\text { Fonctionnement } \\
\text { sexuel }\end{array}$ & 60,0 & 53,0 \\
\hline$*$ (Fitch, Gray et Franssen, 2000) \\
$* *$ (Fitch, Johnson et al., 1999)
\end{tabular}


trouvent que les contextes hospitalier et clinique sont accablants et qu'essayer de comprendre le langage des soins de santé (les mots que les professionnels de la santé utilisent tous les jours) est un défi de taille. Les patients déclarent qu'ils ont souvent de la difficulté à obtenir des renseignements pertinents concernant leur maladie et à trouver des professionnels de la santé qui les aideront à leur expliquer leur propre situation (Fitch, Nicoll et Keller-Olman, 2004). De récents rapports démontrent que les patients atteints d'un cancer ne sont pas satisfaits des renseignements qu'ils reçoivent (voir le tableau 4) et trouvent qu'il s'agit d'un des domaines les plus problématiques du domaine des soins (Fitch et Green, 2005).

Lorsque les individus n'ont pas d'information, ils peuvent ressentir de l'incertitude ainsi qu'une anxiété ou une frustration accrue (Salminea, Vive, Poussa et Knifsund, 2004; Davidson, Degner et Morgan, 1995). Un manque de renseignements pertinents signifie qu'ils sont dans l'impossibilité de prendre part au processus décisionnel en tant que participants informés. Si une personne sait à quoi s'attendre, elle est dans une meilleure position pour se préparer aux éventualités ou choisir parmi les options disponibles celles qui sont le plus en accord avec ses valeurs et ses buts personnels.

À mesure que l'heure du décès approche, beaucoup de patient et de membres de la famille ont des questions sur le processus du mourir et la mort elle-même. Certains auront entendu parler de l'expérience des autres et se demandent si la leur sera identique. Les patients et les membres de la famille se sentiraient peut-être mieux préparés s'ils pouvaient discuter des changements physiques anticipés et de ce à quoi ils peuvent s'attendre.

\section{Les besoins pratiques}

Une fois que nous avons ramené papa à la maison, c'était formidable pour lui. Je le voyais bien. Mais cela signifiait qu'il y avait tant de choses à faire. Je veux dire, si nous sortions tous, nous devions trouver une personne de compagnie. On ne pouvait le laisser seul dans la maison. (fille d'environ 45 ans d'un homme atteint d'un cancer gastro-intestinal)

Tableau 3. Proportion de patients atteints de cancer qui indiquaient que l'information est importante

\begin{tabular}{|l|l|l|}
\hline & \multicolumn{2}{|l|}{ Proportion de patients } \\
\hline Type d'information & $\begin{array}{l}\text { Patientes atteintes } \\
\text { d'un cancer de } \\
\text { l'ovaire N=315* }\end{array}$ & $\begin{array}{l}\text { Patients atteints } \\
\text { d'un cancer de } \\
\text { la prostate } \\
\text { N=621** }\end{array}$ \\
\hline Condition médicale & 88 & 80 \\
\hline $\begin{array}{l}\text { Effets physiques } \\
\text { du cancer }\end{array}$ & 83 & 72 \\
\hline $\begin{array}{l}\text { Choix en matière } \\
\text { de traitement }\end{array}$ & 81 & 72 \\
\hline $\begin{array}{l}\text { Effets secondaires } \\
\text { du traitement }\end{array}$ & 79 & 57 \\
\hline $\begin{array}{l}\text { Façons de soulager } \\
\text { l'inconfort physique }\end{array}$ & 74 & 45 \\
\hline Réactions émotionnelles & 64 & 45 \\
\hline $\begin{array}{l}\text { Où se procurer } \\
\text { fournitures et matériel }\end{array}$ & 48 & 30 \\
\hline $\begin{array}{l}\text { Services de } \\
\text { counseling disponibles }\end{array}$ & 48 & 33 \\
\hline$*$ (Fitch, Gray, DePetrillo, Franssen et Howell, \\
$* *$ (Fitch, Johnson et al., 1999 )
\end{tabular}

Beaucoup de considérations pratiques surgissent lors d'un épisode de maladie (Société canadienne du cancer, 2004; Sanson-Fisher, Girgis, Boyes, Bonevski, Burton et Cook, 2000; Ashbury et al., 1998). Il peut s'agir de considérations à propos du travail, de l'école, des finances, de la garde d'enfants ou des services aux aînés ou des responsabilités du foyer. La maladie signifie souvent que la personne ne peut remplir ses obligations habituelles. Des mesures de rechange doivent être mises en place et peuvent exiger le transfert vers autrui, de responsabilités majeures. À mesure que les limitations fonctionnelles des individus augmentent, de nombreux détails concernant les soins doivent être organisés.

Lorsque la maladie progresse et que la mort est inévitable, plusieurs enjeux pratiques majeurs apparaissent. Une décision pratique importante que certains individus devront prendre est l'emplacement de leur propre décès : à domicile ou dans un établissement. Rester à la maison le plus longtemps possible et mourir dans ce contexte, si le patient le désire, requiert une imposante planification et un accès à un bon service de soutien à domicile. L'emplacement du décès est une décision qui demande maintes réflexions de la part du patient et des prestataires de soins. Les deux contextes possèdent des avantages et des inconvénients. Par exemple, dans un contexte hospitalier, les soins sont prodigués 24 heures sur 24 par le personnel infirmier tandis qu'au domicile, les infirmières peuvent ne visiter qu'une ou deux fois par jour et c'est sur la famille que pèse en grande partie la dispensation des soins; à l'hôpital, la personne peut se sentir davantage séparée de sa famille tandis qu'à la maison, elle peut faire partie du foyer (Edmonds et al., 2001; Grande et al., 1997).

Deux autres enjeux pratiques qui peuvent survenir alors que la maladie progresse incluent la préparation d'un testament ou d'un plan successoral ainsi que les arrangements funéraires. Rédiger un testament permet à une personne de planifier, de façon certaine, la manière dont ses biens seront répartis à la suite de son décès. Cela

Tableau 4. Proportion de patients atteints de cancer non satisfaits de l'information qu'ils avaient reçue

\begin{tabular}{|l|l|l|}
\hline & \multicolumn{2}{|l|}{ Proportion de patients } \\
\hline Type d'information & $\begin{array}{l}\text { Patientes atteintes } \\
\text { d'une récidive du } \\
\text { cancer de l'ovaire } \\
\text { N=95* }\end{array}$ & $\begin{array}{l}\text { Patients atteints } \\
\text { d'une récidive } \\
\text { du cancer de la } \\
\text { prostate N=120** }\end{array}$ \\
\hline Condition médicale & 15 & 25 \\
\hline $\begin{array}{l}\text { Effets physiques } \\
\text { du cancer }\end{array}$ & 18 & 31 \\
\hline $\begin{array}{l}\text { Choix en matière } \\
\text { de traitement }\end{array}$ & 17 & 19 \\
\hline $\begin{array}{l}\text { Effets secondaires } \\
\text { du traitement }\end{array}$ & 10 & 21 \\
\hline $\begin{array}{l}\text { Façons de soulager } \\
\text { l'inconfort physique }\end{array}$ & 12 & 24 \\
\hline $\begin{array}{l}\text { Réactions } \\
\text { émotionnelles }\end{array}$ & 44 & 24 \\
\hline $\begin{array}{l}\text { Où se procurer } \\
\text { fournitures et matériel }\end{array}$ & 12 & 19 \\
\hline $\begin{array}{l}\text { Services de } \\
\text { counseling disponibles }\end{array}$ & 20 & 20 \\
\hline$*$ (Fitch, Gray, DePetrillo, Franssen et Howell, 1999) \\
$* *$ (Fitch, Johnson et al., 1999)
\end{tabular}


pourra également alléger les inquiétudes de la personne sur la façon dont la famille se débrouillera après son décès. La planification des arrangements funéraires avant le décès peut aider la personne à décider de la disposition de son corps et du déroulement de la cérémonie funèbre proprement dite.

\section{Les besoins psychologiques}

J'ai trouvé cela difficile de réaliser que je n'avais pas le contrôle. Je pensais que je l'avais, avant cette maladie. Je prenais des décisions. Je planifiais mes activités. Je faisais des listes. Maintenant, tout est différent. (une patiente atteinte d'un cancer de l'ovaire, 48 ans)

Tout au long d'une maladie, il peut il y avoir différents impacts psychologiques (Ridner, 2004; Farrell et Lewis, 2000; Vachon, 1998). La maladie s'accompagne souvent d'un sentiment de vulnérabilité personnelle et de perte de contrôle sur la vie. Les patients indiquent qu'ils ont l'impression que les événements se produisent autour d'eux et qu'ils se sentent emportés par eux, sans y participer réellement. Certains d'entre eux trouvent qu'il y a une ressemblance avec les montagnes russes avec les arrêts soudains, les plongeons et les virages à grande vitesse qui les caractérisent. Les individus trouvent que leur corps ne fonctionne plus comme avant et qu'ils ne peuvent pas faire ce qu'ils veulent. Non seulement leur image corporelle subit des changements, mais encore leur concept de soi, leur identité personnelle, et leur estime de soi peuvent s'altérer. Lorsqu'une personne ne peut satisfaire ses propres attentes, ne peut réaliser les objectifs qui lui sont chers ou ne peut entretenir de relations importantes, il peut s'ensuivre une détresse psychologique (voir le tableau 5).

La détresse psychologique peut se manifester par de l'anxiété ou de la dépression et peut être ressentie à différents degrés. Jusqu'à un certain point, tous les mourants manifestent un certain niveau d'anxiété. Toutefois, l'anxiété peut varier de faible, à modérée, à sévère (Zabora, Brintzenhofeszoc, Curbow, Hooker et Piantadosi, 2001). Il est important pour tous de pouvoir se confier à quelqu'un et discuter de ce qui nous arrive. Mais les patients aux prises avec les formes modérées et sévères auront avantage à consulter un professionnel en santé mentale (p. ex. un travailleur social, une psychologue) (National Comprehensive Cancer Network, 2002). Environ $25 \%$ seulement des patients manifesteront une dépression clinique bien que d'autres puissent ressentir à l'occasion des sentiments de découragement ou d'impuissance. Les patients les plus à risque d'une dépression sont ceux atteints d'un cancer avancé, qui ont des antécédents de troubles psychologiques ou d'alcoolisme, qui ont une déficience physique accrue, qui ont une douleur incontrôlée, qui ont des maladies concomitantes produisant des symptômes dépressifs, qui prennent certains médicaments et qui manquent de soutien social (Massie, Gagnon et Holland, 1994).

\section{Les besoins spirituels}

Avant je pensais que rien ne pouvait me toucher. Rien ne pouvait m'arriver. Mais cette maladie, elle a tout changé. Je ne suis pas invincible. (une patiente atteinte d'un cancer du sein, 58 ans)

Les maladies à issue mortelle remettent en question nos croyances spirituelles. Apprendre que l'on souffre d'une maladie menaçant le pronostic vital amène souvent des questions : Pourquoi moi? Pourquoi maintenant? Pourquoi de cette façon? Elle menace l'espoir et motive les patients à trouver une signification à la situation ou d'éclairer cette dernière. Elle stimule le désir d'amour et d'appartenance, menace les principales croyances sur l'univers, sur son fonctionnement et la place de chacun en son sein, et nous encourage à regarder au-delà. La « spiritualité » fait référence à la dimension qui motive la raison d'être : la création et l'autotranscendance de la connectivité avec soi-même et avec autrui, y compris un être suprême (Taylor, 1998). C'est la dimension qui nous pousse à clarifier notre conception de l'univers tel qu'on le connaît et à entretenir une relation harmonieuse avec la nature, avec soi-même et avec les autres. Inversement, la religiosité représente une spiritualité plus étroite d'une vision spécifique du monde.

Lorsque les patients luttent avec les enjeux spirituels, ces derniers sont souvent reconnus à tort comme étant des enjeux psychologiques ou émotionnels (p. ex. la peur, l'anxiété, la tristesse, la culpabilité, le désespoir, l'évaluation négative de l'avenir, la mention de la foi, l'agitation). Les enjeux spirituels peuvent se manifester lorsque le patient pose des questions telles que « Pourquoi cela m'arrive-t-il à moi? ». Il peut également exprimer ces besoins en se sentant coupable de s'être éloigné de son groupe confessionnel ou de ses pratiques religieuses.

Trouver des moyens de garder espoir et de donner un sens à la vie est un enjeu important pour ceux qui font face à la mort (Schlesselman, 1998). Même s'il n'y a plus d'espoir réel de guérison, l'espoir peut s'exprimer par le désir d'atteindre des objectifs à court terme (p. ex., une nuit sans douleur, une bonne nuit de sommeil, une mort sans complications, la résolution d'un conflit). Il peut être bénéfique de recadrer l'espoir en mettant l'accent sur le présent et sur des détails particuliers, plutôt que sur de vagues incertitudes (Schlesselman, 1998). L'espoir fait naturellement partie de l'existence humaine et il est reconnu qu'il nourrit les individus accablés de difficultés et sert de tampon contre le stress (Post-White, Ceronsky, Kreitzer, Nickelson, Drew, MacKay et al., 1996). Il est défini comme étant une puissance intérieure dynamique qui permet de transcender une situation actuelle et stimule une nouvelle prise de conscience positive de la raison d'être. On le voit également comme une croyance basée sur l'existence d'un futur positif, mais où l'on admet la possibilité omniprésente de la mort.

Un cancer de stade avancé amène souvent les gens à se questionner sur la signification réelle de leur vie et à déterminer s'ils laissent un héritage. Ils se demandent ce qui va arriver à leurs proches, à l'œuvre de toute leur vie et aux grands buts de leur existence. Au fur et à mesure que les individus passent leur vie en revue, ils peuvent acquérir une connaissance approfondie d'eux-mêmes et de certains

Tableau 5. Questions psychosociales fréquemment soulevées par les patients atteints de cancer

\begin{tabular}{|l|l|l|}
\hline & \multicolumn{2}{|l|}{$\begin{array}{l}\text { Proportion de patients } \\
\text { signalant la question* }\end{array}$} \\
\hline Question & $\begin{array}{l}\text { Patients atteints } \\
\text { d'un cancer du } \\
\text { poumon N=86 }\end{array}$ & $\begin{array}{l}\text { Patients en soins } \\
\text { palliatifs N=63 }\end{array}$ \\
\hline $\begin{array}{l}\text { Craintes relatives à la } \\
\text { propagation du cancer }\end{array}$ & 58 & 36 \\
\hline $\begin{array}{l}\text { Craintes relatives à la } \\
\text { récidive du cancer }\end{array}$ & 49 & 20 \\
\hline $\begin{array}{l}\text { Craintes relatives } \\
\text { à la douleur }\end{array}$ & 41 & 37 \\
\hline $\begin{array}{l}\text { Craintes relatives } \\
\text { à la déficience/ } \\
\text { détérioration physique }\end{array}$ & 34 & 42 \\
\hline $\begin{array}{l}\text { Abattement/ } \\
\text { dépression }\end{array}$ & 40 & 39 \\
\hline Anxiété & 33 & 34 \\
\hline $\begin{array}{l}\text { Désarroi « Pourquoi } \\
\text { cela m'est-il arrivé } \\
\text { à moi? » }\end{array}$ & 19 & 25 \\
\hline$*$ (Fitch et Steele, 2003) & \\
\hline
\end{tabular}


événements et relations qui se sont produits (Holloway et Wheeler, 1996). Cela peut favoriser la croissance personnelle et l'atteinte d'un profond sentiment de paix. Cela peut également être un moment où les individus ressentent une forte motivation à résoudre des affaires ou des enjeux laissés en suspens. Les patients peuvent avoir besoin d'aide pour accomplir ces derniers objectifs importants.

\section{Les besoins sociaux}

Lorsque vous êtes malade, vous voyez vraiment qui sont vos amis, et qui va vous aider. J'ai été surprise et déçue de ce qui est arrivé. Surprise des personnes qui m'ont aidée et déçue de celles qui ne peuvent même pas en parler. (une survivante du cancer du sein, 52 ans)

Le soutien social est un élément-clé de la capacité d'un individu à s'adapter à une situation stressante. Il s'agit de la nature des interactions ou des échanges dans les relations sociales et à l'évaluation qu'en fait le sujet (Stewart, 1993; Sherbourne et Stewart, 1991). En règle générale, on considère que le soutien social possède quatre attributs : instrumental (biens et services tangibles), émotionnel (affection, empathie, amour, confiance), informationnel (faits, résolution de problèmes, rétroaction sur les résultats), et évaluatif (affirmation, approbation). Un ou plusieurs de ces attributs peuvent être menacés lors d'une maladie (Burns, Craft et Roder, 2005). Par exemple, si le travail est la principale voie vers le soutien social et que soudainement la personne ne peut plus travailler, cette voie peut être perdue. Être dans l'impossibilité de prendre part à des activités sociales normales peut signifier une baisse du niveau de soutien social ressenti par la personne.

Le soutien social provient de l'interaction avec les membres de la famille, les amis, les pairs et les prestataires de soins. Une personne qui a le sentiment d'avoir un bon soutien social éprouvera probablement moins d'anxiété et de dépression et pourra voir la situation d'une manière plus positive. Les personnes qui ont un confident, ou même seulement quelqu'un en qui elles croient pouvoir se confier, signalent moins de détresse émotionnelle (Maunsell, Brisson et Deschenes, 1995). Pour les prestataires de soins, il est

Tableau 6. Proportion de patients atteints de cancer qui ont signalé leur crainte de la mort/du mourir

\begin{tabular}{|c|c|}
\hline Groupe de patients & $\begin{array}{l}\text { Proportion signalant } \\
\text { leur crainte de la } \\
\text { mort/du mourir }\end{array}$ \\
\hline $\begin{array}{l}\text { Patientes atteintes d'un } \\
\text { cancer de l'ovaire * } \\
\text { Maladie récidivante }(\mathrm{n}=93) \\
\text { Maladie non récidivante }(\mathrm{n}=170) \\
\text { Patientes atteintes d‘un cancer } \\
\text { gynécologique }(\mathrm{n}=103)^{* *}\end{array}$ & $\begin{array}{l}84 \% \\
33 \% \\
66 \%\end{array}$ \\
\hline $\begin{array}{l}\text { Patients atteints d'un cancer } \\
\text { de la prostate*** } \\
\text { Maladie récidivante }(n=120) \\
\text { Maladie non récidivante }(n=845)\end{array}$ & $\begin{array}{l}18 \% \\
13 \%\end{array}$ \\
\hline $\begin{array}{l}\text { Patients recevant une } \\
\text { irradiation palliative } * * * *(n=63)\end{array}$ & $38 \%$ \\
\hline $\begin{array}{l}\text { Patients atteints d'un cancer du } \\
\text { poumon - divers stades } * * * *(\mathrm{n}=86)\end{array}$ & $37 \%$ \\
\hline $\begin{array}{l}\text { Patients cancéreux }- \text { divers } \\
\text { stades/tous les sites } * * * * *(n=457)\end{array}$ & $53 \%$ \\
\hline \multicolumn{2}{|c|}{$\begin{array}{l}* \text { (Fitch, Gray et Franssen, 2000) } \\
* * \text { (Fitch, Steele, Alderson, Barrow et Constantine, 2004) } \\
* * * \text { (Fitch, Johnson et al., 1999) } \\
* * * * \text { (Fitch et Steele, 2003) } \\
* * * * * \text { (Fitch, Vachon, Greenberg et Franssen, 1996) }\end{array}$} \\
\hline
\end{tabular}

important de reconnaître qu'une relation ne signifie pas toujours que la personne est effectivement soutenue ou se sent soutenue. Certaines relations sont stressantes et exigeantes. Il y a un grand écart dans la qualité du soutien fourni au sein des divers types de relations (p. ex. le mariage, la relation parent-enfant). De plus, ce n'est pas tant le nombre de relations qui compte dans l'existence d'un soutien, mais bien la qualité des relations en jeu.

Des enjeux et des défis peuvent survenir à mesure que la maladie s'aggrave : les relations changent et les membres de la famille et les amis éprouvent un profond chagrin face aux pertes imminentes. Le patient et les membres de la famille peuvent amorcer le processus de deuil avant le décès, appelé deuil anticipé (Loney, 1998). On observe d'ailleurs bon nombre des signes du deuil chez la personne qui fait son deuil de la perte imminente, avant la survenue du décès. Les membres de la famille se préparent de plusieurs façons à vivre sans le mourant. Ils sentent que le monde change irrévocablement et que les choses ne seront plus jamais les mêmes. Imaginer la vie sans un être cher peut faire naître, chez un membre de la famille, des sentiments accablants de désespoir, d'impuissance et de perte profonde, et peut empêcher celui-ci d'offrir un soutien au mourant.

\section{Les besoins émotionnels}

J'ai de bonnes journées et de moins bonnes journées. Je suis heureuse de certaines choses, et triste d'autres. J'aimerais pouvoir changer les choses et ne pas avoir cette maladie, mais je ne peux pas. (une femme atteinte de myélome multiple, 56 ans)

Faire face à sa propre mort suscite diverses émotions (Association canadienne d'oncologie psychosociale, 2003). Chaque personne réagira à sa façon. Il peut y avoir un choc et de l'incrédulité que cela puisse arriver, de la colère que cela arrive à ce moment de la vie, ou l'acceptation que l'heure ait sonné. Toute une gamme d'inquiétudes fait habituellement surface lorsque la personne commence à imaginer la fin de sa vie et ce qui arrivera aux êtres qu'elle aime. Au fur et à mesure qu'il y a des changements physiques et que la personne devient de plus en plus dépendante des autres, elle peut s'inquiéter d'être un fardeau et de ne plus contribuer à la famille. Lorsqu'une personne n'est plus capable de faire quelque chose qu'elle pouvait normalement faire, elle peut ressentir de la frustration. Des regrets à propos de choses qui ont été faites ou, au contraire, non faites, peuvent entraîner des sentiments de culpabilité ou de remord.

De nombreuses peurs peuvent faire leur apparition à mesure que la maladie évolue. Des patients peuvent craindre le futur inconnu ou craindre la mort elle-même (voir le tableau 6). Ils peuvent craindre une mort douloureuse et inconfortable ou encore mourir seuls. Certains ont peur d'être abandonnés par leur famille, leurs amis ou même les professionnels de la santé. D'autres ont peur de perdre le contrôle de leur corps, leur esprit, leur capacité de prendre des décisions, leur vie et la manière dont ils passeront leurs derniers jours.

Pour certains individus, les émotions peuvent être si accablantes et effrayantes que le meilleur moyen de faire face à la situation est de les mettre de côté et de continuer comme si de rien n'était. Lorsque c'est le cas, on dit que les patients sont en déni. Toutefois, utiliser le déni à l'occasion peut constituer une stratégie très efficace pour s'adapter à une situation (Gorman, 1998). Le déni peut aider une personne à maîtriser ses émotions lorsqu'elles menacent de l'accabler. Il peut également être utile comme stratégie d'adaptation lorsqu'il n'y a plus rien à faire pour changer l'issue d'une situation. Lorsqu'une personne se retrouve dans cette situation, il est parfois plus simple d'éviter de s'arrêter aux résultats indésirables du futur et de concentrer son énergie sur le présent et ce qui peut y être accompli. Il est important de voir qu'il n'y a pas de « bonne façon » de s'adapter à ce genre de situation et qu'il n'y a pas d'échéancier « établi » pour les sentiments.

Chez les personnes atteintes d'un cancer avancé dont la mort est imminente, chaque journée est une recherche d'équilibre - l'équilibre entre la vie et la mort - et un face à face avec l'incertitude. C'est une période d'émotions intenses pour plusieurs personnes. Les histoires 
des patients vivant ces moments sont remplies de courage, de désespoir, d'espoir et de chaos. Les citations suivantes, dont les auteurs étaient sur le point de mourir, illustrent bien ces points :

"Cette maladie a complètement anéanti presque tous mes rêves et mes espoirs. Parce qu'il n'y a pas de traitement efficace contre cette maladie, mon espérance de vie est très courte. Je ne verrai pas mes enfants s'épanouir pour devenir des adultes. Je ne partagerai pas les années à venir avec mon mari. Je ne suis pas capable d'entreprendre des activités physiquement ardues avec mes amis et ma famille. Ceci est particulièrement difficile parce que mes enfants sont encore jeunes. Je ne peux plus travailler, ce qui a changé notre situation financière. Mais ce qui importe le plus, à mes yeux, est de savoir que mes enfants n'auront bientôt plus de mère. " (une patiente atteinte d'un cancer de l'ovaire, 32 ans)

"Je regarde la vie d'une façon plus positive et j'apprécie davantage les choses et la vie. Notre vie spirituelle s'est approfondie. Je prends le temps de sentir les roses. Les détails qui me semblaient tellement importants ne le sont plus... je laisse la vaisselle traîner dans l'évier pendant que je fais une promenade... je suis moins pressée de tout accomplir. " (une femme atteinte d'un cancer colorectal, 68 ans)

"Je ne peux plus sortir autant qu'avant. Mais on ne me laisse jamais seule très longtemps. Je me sens vraiment piégée et je n'ai plus de vie du tout maintenant... Il y a beaucoup de choses que j'aimerais faire, mais que je ne peux plus faire. Je ne peux plus me permettre de faire les choses que je voulais faire pour me simplifier la vie. Les gens m'évitent. " (une femme atteinte d'un cancer du poumon, 42 ans)

"Ma vie est complètement différente maintenant. J'ai besoin d'un long repos tous les après-midi alors il ne reste plus beaucoup de temps dans la journée pour accomplir les activités de base. Toutefois, je chéris les visites de mes amis et les moments où je leur parle au téléphone. Lorsque mon mari m'emmène faire un tour en voiture, j'apprécie davantage la nature que je vois autour de moi. Je considère que j'ai encore une bonne qualité de vie malgré les pertes que j'ai subies. " (une patiente atteinte d'un cancer de l'ovaire, 71 ans)

\section{Les approches d'intervention}

Les infirmières qui soignent les patients atteints d'un cancer avancé doivent d'abord reconnaître qu'il n'y a pas deux individus qui vont vivre et réagir à une situation particulière exactement de la même façon (Lazarus et Folkman, 1984). Les individus réagissent selon la perception qu'ils ont de la situation dans laquelle ils se trouvent. Leur interprétation du préjudice ou de la menace imminent(e) causée par l'événement/la situation, envers eux-mêmes et envers les êtres qui leur sont chers, déterminera les approches émotionnelles, cognitives et comportementales auxquelles ils feront appel pour s'adapter à la situation. Les infirmières peuvent effectuer une évaluation approfondie en utilisant des questions fermées et ouvertes, afin de comprendre la perspective unique de chaque patient (Nicholas et Veach, 2000). Il faudra probablement assez de temps pour créer une relation de confiance afin que la personne puisse confortablement parler de sujets délicats et difficiles, et l'infirmière ne devra porter aucun jugement et devra chercher à comprendre les situations et les événements selon la perspective du patient. L'infirmière doit essayer de comprendre les menaces, les pertes et les défis tels qu'ils sont perçus par la personne. Un défi de taille pour beaucoup d'infirmières est de s'abstenir de porter un jugement face à la perspective et aux stratégies d'adaptation que le patient utilise dans une situation particulière, lorsque ces dernières ne sont pas celles que les infirmières utiliseraient. Il est primordial que les infirmières soient capables de mettre de côté leurs idées et leurs points de vue personnels sur la manière de s'adapter à une situation et de ne pas les confondre avec les approches que le patient utilise. Une chose qui s'avère efficace et utile pour l'un ne l'est pas nécessairement pour l'autre.
Lors de leurs conversations avec un patient, les infirmières doivent cerner les buts et les valeurs qui lui sont chers. Comprendre ce qui est important pour l'individu fournit un cadre de référence permettant de planifier ce qui devrait être fait, de dégager les résultats importants pour les interventions, et de déterminer les critères d'évaluation du succès. Les désirs ou les vœux du patient doivent être la considération primordiale. Des tensions peuvent naître si l'infirmière n'a pas les mêmes buts que le patient. Par exemple, l'infirmière peut penser que tous les membres de la famille devraient être informés de la mort imminente du patient, mais celuici s'y oppose peut-être; ou un patient peut souhaiter arrêter tout apport nutritionnel alors que le professionnel de la santé veut continuer l'alimentation par voie intraveineuse; ou un patient ne veut pas prendre la dose complète de son analgésique pour demeurer alerte, alors que le professionnel de la santé pense que la dose complète est requise pour maîtriser la douleur.

Il y a deux buts principaux lorsqu'on intervient auprès de patients atteints d'un cancer avancé : 1) diminuer l'impact des symptômes et des effets secondaires; et, 2) aider l'individu à s'adapter à l'impact de la situation (Fitch, 2003). Pour atteindre ces buts, les interventions doivent être adaptées et individualisées. Pour connaître le meilleur moyen d'adapter des interventions pour une personne, on doit avoir une conversation avec cette dernière à propos de ses voeux et de son évaluation de la situation (Lazarus et Folkman, 1984). La conversation doit se concentrer sur des enjeux tels que le niveau d'implication désiré de l'individu dans le processus décisionnel relatif aux soins et de quels renseignements il a besoin pour participer de la façon désirée. On doit offrir à l'individu plusieurs occasions d'exprimer ses inquiétudes ou de poser des questions sans craindre qu'on le juge ou qu'on fasse fi des inquiétudes exprimées. Ces interactions avec le patient et les membres de la famille devraient être régulières. En effet, les situations changent au fil du temps et les gens changent d'idées. Sans des conversations régulières, l'équipe soignante peut ne pas dispenser des soins réellement axés sur le patient.

La myriade d'enjeux qui peut survenir chez le mourant et les membres de la famille exige que l'on travaille au sein d'une équipe interdisciplinaire. Bien qu'un membre de l'équipe puisse avoir un contact privilégié avec le patient (il peut s'agir du médecin ou de l'infirmière), on peut faciliter l'accès à d'autres disciplines. Selon la nature de l'enjeu vécu par le patient, ce dernier pourrait avoir besoin de consulter un membre d'une autre discipline, ou ce professionnel pourrait être consulté par le médecin ou l'infirmière du patient afin d'obtenir des conseils.

Certains auteurs recommandent d'utiliser des listes de contrôle des symptômes normalisées (Sarna, 1998) ou des instruments de dépistage psychosocial (Zabora et al., 2001). Ces outils simplifient l'évaluation et la documentation routinières des symptômes physiques et psychologiques. De plus, l'application de ces outils favorise une surveillance continue de l'efficacité des interventions et permet les consultations, en temps opportun, auprès d'autres disciplines.

\section{Résumé}

Se trouver confronté à sa propre mort ou à celle d'un membre de la famille est difficile. Face à cette situation, la plupart des personnes éprouvent une myriade d'émotions. Bien que certaines les gèrent remarquablement bien, d'autres ont besoin d'une aide spécialisée. Pour prodiguer des soins efficaces, il est très important d'adapter les interventions en fonction des perspectives et des buts uniques du patient. Il est primordial d'établir une relation de confiance avec chaque patient si l'on veut mieux comprendre ses buts et ses valeurs. Lorsque les infirmières prennent en charge des individus atteints d'un cancer avancé, il est essentiel que les buts et les valeurs de ces derniers soient au coeur du processus de planification des soins. 


\section{Références}

Ashbury, F.D., Findlay, H., Reynolds, B., \& McKerralehen, K.A. (1998). Canadian survey of cancer patients' experiences: Are their needs being met? Journal of Pain and Symptom Management, 6(5), 298-306.

Burns, C.M., Crafts, P.S., \& Roder, D.M. (2005). Does emotional support influence survival? Findings from a longitudinal study of patients with advanced cancer. Supportive Cancer Care, 13, 295302 .

Association canadienne d'oncologie psychosociale. (2003). Vivre avec le cancer - Un bouleversement affectif - Un guide de soutien aux personnes atteintes de cancer et leurs proches. Calgary. Auteur.

Davidson, B.J., Degner, L.F., \& Morgan, T.R. (1995). Information and decision-making preferences of men with prostate cancer. Oncology Nursing Forum, 22(9), 1401-8.

Edmonds, P., Karlsen, S., Khan, S., \& Addington-Hall, J. (2001). A comparison of the palliative care needs of patients dying from chronic respiratory diseases and lung cancer. Palliative Medicine, 15(4), 287-95.

Farrell, C., \& Lewis, J. (2000). The Cancer Experience. London: National Centre for Social Research.

Ferrell, B.R. (1996). The quality of lives: 1525 voices of cancer. Oncology Nursing Forum, 23, 909-916.

Fitch, M.I., Vachon, M., Greenberg, M., \& Franssen, E. (1996). Needs of cancer patients and their family members attending a comprehensive cancer centre. Proceedings of the 9th International Nurses in Cancer Care Conference; Aug 12-15, Brighton, UK. p. 68.

Fitch, M.I., Johnson, B., Gray, R.E. et Franssen, E. (1999). L'impact du cancer de la prostate du point de vue des survivants: répercussions pour les soins infirmiers en oncologie. Revue canadienne de soins infirmiers en oncologie, 9(1), 29-34.

Fitch, M.I., Gray, R.E., DePetrillo, D., Franssen, E., \& Howell, D. (1999). Canadian women's perspectives on ovarian cancer. Cancer Prevention \& Control, 3(1), 52-60.

Fitch, M.I., (2000). Supportive Care for Cancer Patients. Hospital Quarterly, 3, 39-46.

Fitch, M.I., Gray, R.E., \& Franssen, E. (2000). Women's perspectives regarding the impact of ovarian cancer. Cancer Nursing, 23(5), 359-366.

Fitch, M.I. (2003). Psychosocial Management of patient with recurrent ovarian disease: Treating the whole person to improve quality of life. Seminars in Oncology Nursing, 19(3), 40-53.

Fitch, M.I., \& Steele, R. (2003). Supportive care needs of cancer patients: Looking beyond the obvious. Supportive Care Cancer, 11(6), 423.

Fitch, M.I., Steele, R., Alderson, D., Barrow, K., Constantine, T., \& Doyle, C. (2004). Understanding supportive care needs of patients with gynecological cancer. Supportive Care Cancer, 12(6), 411.

Grande, G.E., Rodd, C.J., \& Barcley, S.I. (1997). Supports needed in the last year of life: Patients and carer dilemmas. Palliative Medicine, 11(3), 202-8.

Gray, R.E., Greenberg, M., Fitch, M.I., Sawka, C., Hampson, A., Lebrecque, M., \& Moore, B. (1998). Information needs of women with metastatic breast cancer. Cancer Prevention \& Control, 2(2), 57-62.

Gorman, L.M. (1998). Denial. In, R.M. Carroll-Johnson, L.M. Gorman, \& W.J. Bush (Eds.), Psychosocial Nursing Care (pp. 171-181). Pittsburgh, PA: Oncology Nursing Press, Inc.

Herth, K. (1993). Hope in the family caregiver of terminally ill people. Journal of Advanced Nursing, 18, 538-48.
Holloway, I., \& Wheeler, S. (1996). Qualitative Research for Nurses. Edinburgh: Blackwell Science.

Lazarus, R.S., \& Folkman, S. (1984). Stress, appraisal and coping. New York: Springer.

Loney, M. (1998). Death, dying and grief in the face of cancer. In E.S. Burke (Ed.), Psychosocial dimensions of oncology nursing care (pp. 151-179). Pittsburg, PA: Oncology Nursing Press, Inc.

Massie, M.J., Gagnon, P., \& Holland, J.C. (1994). Depression and suicide in patients with cancer. Journal of Pain and Symptom Management, 9, 325-340.

Maunsell, E., Brisson, J., \& Deschenes, L. (1995). Social support and survival among women with breast cancer. Cancer, 76, 631637.

McLean, G.L. (1993). Facing death: Conversations with cancer patients. London: Churchill Livingstone.

National Comprehensive Cancer Network. (2002). NCCN Practice guidelines in oncology: Distress management. Retrieved July 3, 2002, from http://www.nccn.org/ physician_gls/index.html

Nicholas, D.R., \& Veach, T.A. (2000). The psychosocial assessment of the adult cancer patient. Professional Psychology Research Practice, 206-15.

Post-White, J., Ceronsky, C., Kreitzer, M.J., Nickelson, K., Drew, D., MacKay, K.W., Koopermeiners, L., et al. (1996). Hope, spirituality, sense of coherence and quality of life with cancer. Oncology Nursing Forum, 23, 1303-1309.

Preston, F.A., \& Cunningham, R.S. (1998). Clinical Guidelines for Symptom Management in Oncology. New York: Clinical Insights Press Inc.

Ridner, S.H. (2004). Psychological distress: Concept analysis. Journal of Advanced Nursing, 45(5), 536-45.

Salminea, E., Vive, J., Poussa, T., \& Knifsund, S. (2004). Unmet needs for information flow between breast cancer patients, their spouses and physicians. Supportive Care Cancer, 12, 663668.

Sanson-Fisher, R., Girgis, A., Boyes, A., Bonevski, B., Burton, L., \& Cook, P. (2000). The unmet needs of patients with cancer. Cancer, 88(1), 217-25.

Sherbourne, C., \& Stewart, A. (1991). The MOS social support survey. Social Science and Medicine, 32(6), 705-714.

Sarna, L. (1998). Effectiveness of structure of nursing assessment of symptom distress in advanced lung cancer. Oncology Nursing Forum, 25(6), 1041-7.

Schlesselman, S.M. (1998). The influence of hope on the psychosocial experience. In R.M. Carroll-Johnson, L.M. Gorman, \& W.J. Bush (Eds.), Psychosocial Nursing Care (pp. 83-89). Pittsburgh, PA: Oncology Nursing Press, Inc.

Singer, P.A., Martin, D.K., \& Kelner, M. (1999). Quality end of life care: Patients' perspectives. Journal of the American Medical Association, 281(2), 163-8.

Stewart, M. (1993). Integrating social support in nursing. Newbury Park, CA: Sage Publications.

Taylor, E.J. (1998). Spirituality and the cancer experience. In, R.M. Carroll-Johnson, L.M. Gorman, \& W.J. Bush (Eds.), Psychsocial Nursing Care (pp. 71-82). Pittsburgh: Oncology Nursing Press, Inc.

Vachon, M. (1998). Psychosocial needs of patients and families. Journal of Palliative Care, 14(3), 49-56.

Zabora, J., Brintzenhofeszoc, K., Curbow, B., Hooker, C., \& Piantadosi, S. (2001). The prevalence of psychological distress by cancer site. Psycho-oncology, 10(1), 19-28. 